\title{
MORFOLOGÍA Y PROTRUSIÓN-RETRACCIÓN DE LA CÁMARA GENITAL FEMENINA DE PHYLLOPHAGA OBSOLETA (COLEOPTERA: MELOLONTHIDAE)
}

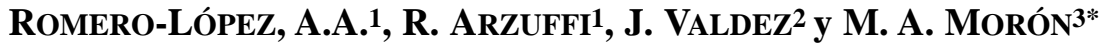 \\ ${ }^{1}$ Centro de Desarrollo de Productos Bióticos, Instituto Politécnico Nacional, Yautepec, Morelos, \\ Código postal 62731, MÉXICO. \\ ${ }^{2}$ Colegio de Postgraduados, Campus Montecillo, Estado de México, Código postal 56230, MÉXICO. \\ 3Instituto de Ecología, A.C. Xalapa, Veracruz, Código postal 91000, MÉXICO. \\ * Autor correspondencia: miguel.moron@inecol.edu.mx
}

Romero-López, A. A., R. Arzuffi, J. Valdez y M. A. Morón. 2009. Morfología y protrusión-retracción de la cámara genital femenina de Phyllophaga obsoleta (Coleoptera: Melolonthidae). Acta Zoológica Mexicana (n. s.), 25(2): 315-321.

RESUMEN. Las hembras de Phyllophaga obsoleta (Blanchard) (Coleoptera: Melolonthidae), una de las especies de mayor importancia agrícola en México, presentan un "llamado sexual" en el cual exponen una estructura en forma de bolsa del ápice abdominal y provocan la atracción de los machos. El contenido de esta estructura se ha extraído en estudios previos con diferentes disolventes y se ha confirmado su actividad biológica mediante bioensayos con túnel de viento y electroantenografía. Sin embargo, las características morfológicas de esta "bolsa" no han sido estudiadas. Por ello, en el presente trabajo se caracterizó morfológicamente los terminalia del abdomen femenino de $P$. obsoleta y se estudió el mecanismo de protrusión-retracción de la estructura en forma de bolsa. Se realizaron disecciones longitudinales de los terminalia del abdomen de hembras para su análisis con microscopía estereoscópica. Se encontró que la "bolsa" es la cámara genital protráctil (CGP), propia de las hembras de Melolonthidae y que se encuentra flanqueada por músculos esterno-saculares laterales y esternosaculares ventrales, así como de membranas intersegmentales VIII y IX. Músculos y membranas podrían estar involucrados en los procesos de protrusión y retracción de la CGP.

Palabras clave: Phyllophaga obsoleta, llamado sexual, cámara genital, protrusión, retracción.

Romero-López, A. A., R. Arzuffi, J. Valdez \& M. A. Morón. 2009. Morphology and protrusionretraction of the female genital chamber of Phyllophaga obsoleta (Coleoptera: Melolonthidae). Acta Zoológica Mexicana (n. s.), 25(2): 315-321.

ABSTRACT. The females of Phyllophaga obsoleta (Blanchard) (Coleoptera: Melolonthidae) have a great agricultural importance in Mexico. They display a "sexual calling" behavior, that expose a bag-like structure on the abdominal tip, wich causes them to attract the males. The content of this structure has been extracted and confirmed his biological activity by wind tunnel and electroantenography bioessays. Nevertheless, their morphologic characteristics have not been studied yet. For that reason in the present

Recibido: 28/02/2008; aceptado: 15/09/2008. 
study, the $P$. obsoleta abdominal terminalia were characterized morphologically and protrusion-retraction mechanism of bag-like structure was studied. Longitudinal dissections of the abdominal terminalia were analyzed with stereoscopic microscopy. The bag-like structure is the protractile genital chamber (PGC) of Melolonthidae females and its protrusion and retraction is related to the activity of lateral sterno-sacular muscles with ventral sterno-sacular muscles, as well as for intersegmental membranes VIII and IX. They should be involucrated in protrusion and retraction PGC mechanisms.

Key words: Phyllophaga obsoleta, calling, genital chamber, protrusion, retraction.

\section{INTRODUCCIÓN}

Existen pocos trabajos acerca de la morfología de las glándulas productoras de feromonas sexuales de coleópteros. Los estudios realizados se limitan a especies que ofrecen interés desde el punto de vista económico (Coca-Abia \& Martin-Piera 1991). En coleópteros de la familia Melolonthidae se han detectado glándulas en donde la feromona es emitida al exterior a través de canales cuticulares (Tada \& Leal 1997) y un epitelio glandular en el interior de una estructura en forma de bolsa que exponen las hembras antes del apareamiento (Kim \& Leal 1999). Esta exposición de una "bolsa" se ha documentado como parte del comportamiento sexual de algunas especies (Leal 1998, 1999, Oliveira \& García 2003; Arakaki et al. 2003). En el caso de Phyllophaga obsoleta (Blanchard), una especie de Melolonthidae de importancia económica en México (Morón 2003), se sabe que su comportamiento sexual se compone de una serie consecutiva de actividades que conducen al apareamiento (Eberhard 1993). El denominado "llamado sexual" es un componente de esta secuencia que involucra una serie de movimientos y posturas por parte de la hembra que concluyen con la exposición de una bolsa; posterior a ello se presenta el acercamiento de los machos y la cópula (Romero-López et al. 2003).

Para los fines del presente estudio, se pretende conocer más sobre la morfología de esta bolsa, además de las estructuras que permiten su protrusión y retracción en el momento del llamado sexual de las hembras de P. obsoleta.

\section{MATERIAL Y MÉTODOS}

Insectos. Los ejemplares de $P$. obsoleta utilizados en este trabajo se capturaron en julio del 2006, en trampas de luz colocadas en el campo experimental del Centro de Desarrollo de Productos Bióticos, ubicado en Yautepec, Morelos, México.

\section{Preparación de material y estudio morfológico de la terminalia abdominal.} Las hembras de P. obsoleta se mantuvieron en etanol al $70 \%$ hasta el momento de la disección y la separación de las estructuras en forma de bolsa. Posteriormente se separaron los segmentos terminales del abdomen, los cuales se colocaron en $\mathrm{KOH}$ al $10 \%$ a $80^{\circ} \mathrm{C}$ durante $20 \mathrm{~min}$; se lavaron con agua destilada y se conservaron en etanol al 70\%. Para la obtención de las imágenes y esquemas de las estructuras a estudiar, se realizaron observaciones en un microscopio estereoscópico modelo Zeiss SV-6, con cámara clara. 


\section{RESULTADOS}

Con las observaciones en el microscopio estereoscópico se determinó que la bolsa que exponen las hembras de P. obsoleta al efectuar su llamado sexual es en realidad un antro, cavidad o cámara revestida de tejido epitelial y paredes musculares que se encuentra en la terminal posterior del octavo segmento abdominal, como una extensión posterior del oviducto. Esta cámara tiene la propiedad de ser protruida o expuesta en determinadas condiciones y por ello, en el presente estudio, se le dio el nombre de cámara genital protráctil (CGP). Se encontró que esta cámara está flanqueada por las membranas intersegmentales VIII y IX que unen al terguito y esternito del segmento VIII con el terguito y esternito del segmento IX (Fig. 1). También se encontraron dos tipos de músculos unidos a la CGP: un par de músculos esterno-saculares laterales y dos pares de músculos esterno-saculares ventrales. Estas láminas musculares delgadas y delicadas se originan en las paredes externas de la CGP y se insertan en la parte interna de la región pigidial (Fig. 2).

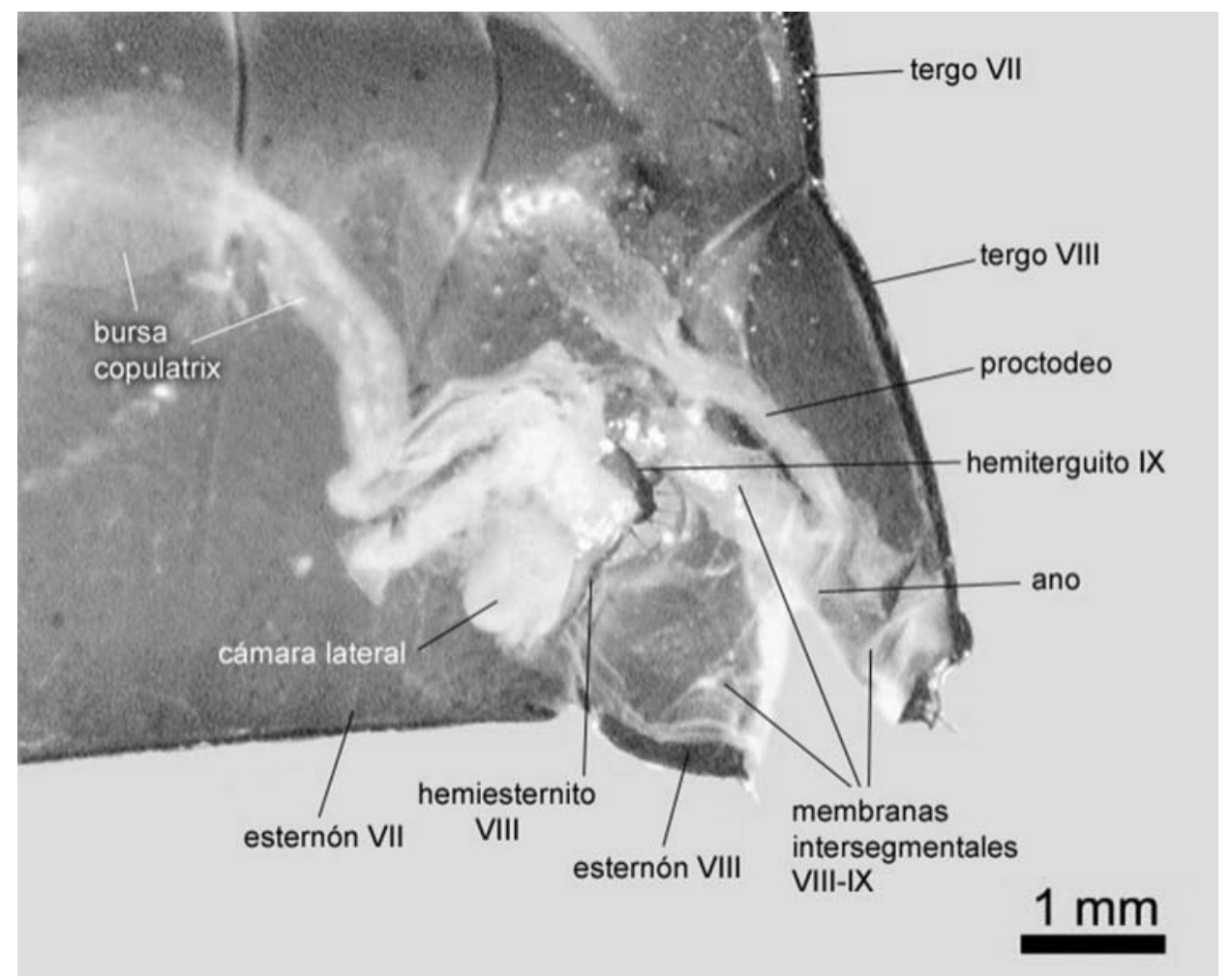

Figura 1. Imagen de los terminalia abdominales de la hembra de Phyllophaga obsoleta, en donde se distinguen las membranas intersegmentales VIII y IX asociadas a la protrusión de la cámara genital. 


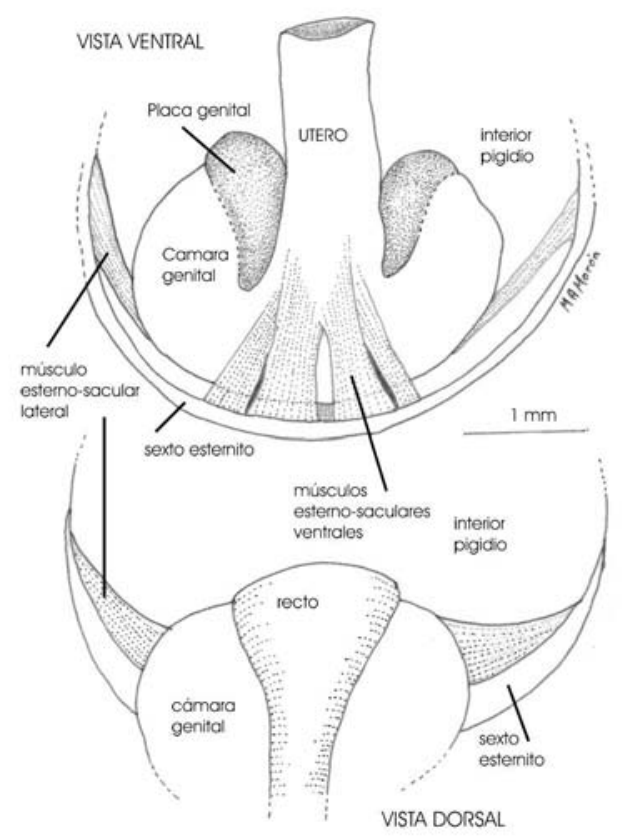

Figura 2. Vista dorsal y ventral de los terminalia abdominales de la hembra de Phyllophaga obsoleta, en donde se muestran los músculos asociados a la protrusión de la cámara genital.

\section{DISCUSIÓN}

La bolsa que exponen las hembras al llamar a los machos es la cámara genital que caracteriza a las hembras de Melolonthidae (Berberet \& Helms 1972, Coca-Abia \& Martín-Piera 1991, Coca-Abia et al. 1993, Krell 1996). El nombre CGP se consideró más apropiado que los términos bolsa, "bola" o "glándula" empleados en estudios previos (Leal 1998, Kim \& Leal 1999, Oliveira et al. 2003), ya que refiere al nombre original de la estructura y su condición de protractilidad.

En general, en este grupo de coleópteros la cámara genital es considerada como la unión del poro genital con invaginaciones membranosas del integumento abdominal (Kim \& Leal 1999), que tiene como una de sus principales funciones la introducción y permanencia del edeago masculino en el momento de la cópula (Berberet \& Helms 1972, Krell 1996). En el caso de las hembras de algunas especies (como $P$. obsoleta), se ha documentado una función secundaria que involucra la exposición de esta estructura para la liberación de sustancias atrayentes en el momento de la actividad sexual (Morón 1986, Leal 1998, Romero-López et al. 2005). Este papel secundario es exclusivo de especies de Phyllophaga, Holotrichia y 
Melolontha, las cuales además de lo anterior, comparten aspectos biológicos, ecológicos y filogenéticos que las relaciona estrechamente (Leal 1998, Ruther et al. 2001, Romero-López 2008). Aunque no se sabe del por qué de esta función secundaria de la CGP, es probable que tenga que ver con la condición propia del comportamiento sexual de estas especies, en donde predomina la atracción de machos que se encuentran relativamente alejados de las hembras, en hábitats con condiciones ambientales diversas (Romero López et al. 2007), ya sea por la necesidad de contar con un referente visual para los machos (la CGP femenina protruida) o para la liberación de las feromonas sexuales a una mayor presión, con el fin de alcanzar distancias más extensas. Esto, a diferencia de lo que sucede con especies del género Anomala en donde la actividad sexual generalmente se desarrolla a una distancia corta entre hembras y machos (Facundo et al. 1999), para lo cual no es necesaria la exposición de la cámara genital y basta con la liberación de feromonas a través de poros cuticulares abdominales (Tada \& Leal 1997).

De acuerdo con observaciones realizadas en hembras de Melolontha melolontha (Linnaeus) (Krell 1996), Holotrichia parallela (Motchulsky) (Kim \& Leal 1999) y Megasoma elephas (Fabricius) (Morón, datos no publicados), se sabía de la participación de membranas intersegmentales y de algunos músculos abdominales en la exposición y retracción de la CGP. En el caso de las hembras de P. obsoleta, se encontró un arreglo de membranas y músculos similar al de las especies mencionadas, sugiriendo una división de trabajo entre ambos tipos de músculos, en paralelo con la acción de las membranas intersegmentales, como han citado Krell (1996) y Kim \& Leal (1999). Con base en estos antecedentes y en las características morfológicas de la CGP y estructuras circundantes ya descritas, en el presente estudio se propone una secuencia de eventos que estarían integrando el mecanismo de protracción-retracción de la CGP. Es probable que cuando la hembra inicia su llamado sexual y expone la CGP, se presenten cinco eventos: a) la contracción y relajación simultánea de los músculos esterno-saculares laterales, provocando los movimientos ondulantes de la región abdominal de la hembra; b) desplazamiento de la hemolinfa hacia la región posterior del insecto; c) salida de la CGP a través de la abertura ano-genital, con la participación de las membranas y los músculos esternosaculares ventrales (acción complementaria de empuje hacia el exterior); d) relajación de ambos tipos de músculos y estiramiento de las membranas para el sostenimiento de la CGP en su momento de protrusión y e) acción simultánea de retraimiento de membranas y contracción de ambos tipos de músculos, jalando hacia al interior la CGP y regresándola a su posición original.

Esta secuencia de eventos podría formar parte del repertorio de patrones de comportamiento de la hembra de $P$. obsoleta. En el momento de su llamado se observa un movimiento rítmico de su abdomen en círculos y de arriba abajo (Romero-López et al. 2003) y aquí podrían estar actuando los músculos laterales, 
como ya se ha descrito. Esto permite sugerir que la exposición de la CGP, además de la influencia de factores ambientales externos, sería el resultado de la acción mecánica de los músculos laterales y ventrales que impulsan el movimiento de la hemolinfa (Kim \& Leal 1999), provocando un efecto similar al de un émbolo.

Con el fin de conocer más acerca de la comunicación química y el comportamiento sexual de $P$. obsoleta, se requiere complementar esta información sobre la morfología externa de la CGP con estudios que permitan determinar la región específica de esta estructura en donde podrían producirse las feromonas sexuales. Para ello se han iniciado los estudios histológicos correspondientes.

AGRADECIMIENTOS. Angel A. Romero es becario IPN y para la realización del presente trabajo recibió apoyo por parte del PIFI; R. Arzuffi es becario EDI y COFAA, IPN.

\section{LITERATURA CITADA}

Arakaki, N., S. Wakamura, H. Yasui, Y. Sadoyama \& M. Kishita. 2003. Sexually differentiated functions of female-produced pheromone of the black chafer Holotrichia loochooana loochooana (Sawada) (Coleoptera: Scarabaeidae). Chemoecology, 13: 183-186.

Berberet, R.C. \& T.J. Helms. 1972. Comparative anatomy and histology of selected systems in larval and adult Phyllophaga anxia (Coleoptera: Scarabaeidae). Annals of Entomological Society of America, 65: 1026-1053.

Coca-Abia, M. \& F. Martín-Piera. 1991. Anatomy and morphology of the genitalia in the subtribe Rhizotrogina (Coleoptera: Melolonthidae: Melolonthini); taxonomic implications. In: M. Zunino, X. Belles y M. Blas (Eds). Advances in Coleopterology. AEC, Barcelona. pp. 61-78.

Coca-Abia, M., F. Martín-Piera \& M.A. Morón. 1993. Anatomía y morfología de la genitalia femenina de las especies del género Phyllophaga (sensu lato) (Coleoptera: Melolonthidae). Relaciones filogenéticas con otros géneros del área mediterránea. Giornale Italiano di Entomologia, 6: 263274.

Eberhard, W. G. 1993. Copulatory courtship and morphology of genitalic coupling in seven Phyllophaga species (Coleoptera: Melolonthidae). Journal of Natural History, 27: 683-717.

Facundo, H.T., C.E. Linn Jr., M.G. Villani \& W.L. Roelofs. 1999. Emergence, mating and postmating behaviors of the oriental beetle (Coleoptera: Scarabaeidae). Journal of Insect Behavior, 12: 175-192.

Kim, J.Y. \& W.S. Leal. 1999. Eversible pheromone gland in a melolonthinae beetle, Holotrichia parallela. Journal of Chemical Ecology, 25: 825-833.

Krell, F.T. 1996. The copulatory organs of the cockchafer Melolontha melolontha (Insecta: Coleoptera: Scarabaeidae). A contribution to comparative and functional anatomy of ectodermal genitalia of the Coleoptera. Stuttgarter Beiträge zur Naturkunde Serie A (Biologie), 537: 1-101.

Leal, W.S. 1998. Chemical ecology of phytophagous scarab beetles. Annual Reviews of Entomology, 43:39-61.

Leal, W.S. 1999. Scarab beetles. Pp: 51-68. In: J. Hardie \& A.K. Minks (Eds.). Pheromones of non-lepidopteran insects associated with agricultural plants. CAB International. Wallingford, UK.

Morón, M.A. 1986. El género Phyllophaga en México (Insecta: Coleoptera). Morfología, distribución y sistemática supraespecífica. Publicación No. 20, Instituto de Ecología, México.

Morón, M.A. 2003. Diversidad, distribución e importancia de las especies de Phyllophaga Harris en México (Coleoptera: Melolonthidae). Pp. 1-27. In: A. Aragón, M.A. Morón \& A. Marín-Jarillo 
(eds.). Estudios sobre coleópteros del suelo en América. Publicación especial de la Benemérita Universidad Autónoma de Puebla, México.

Oliveira, L.J. \& M.A. García. 2003. Flight, feeding and reproductive behavior of Phyllophaga cuyabana (Moser) (Coleoptera: Melolonthidae) adults. Pesquisa agropecuária brasileira, 38: 179-186.

Romero-López, A.A. 2008. Comunicación química sexual de Phyllophaga obsoleta (Coleoptera: Melolonthidae). Tesis de Doctorado (inédita), CEPROBI, Instituto Politécnico Nacional.

Romero-López, A.A., A. Aragón \& R. Arzuffi. 2007. Estudio comparativo del comportamiento sexual de cuatro especies de Phyllophaga (Coleoptera: Melolonthidae). 275-281 pp. In: E. G. Estrada, A. Equihua, C. Luna y J.L. Rosas-Acevedo (Eds.). Entomología mexicana, Vol. 6. Sociedad Mexicana de Entomología. México.

Romero-López, A.A., R. Arzuffi, F.J. Villalobos \& M.A. Morón. 2003. Comportamiento sexual de Phyllophaga obsoleta (Coleoptera: Melolonthidae). pp. 197-202. In: J.E. Romero-Nápoles, E. G. Estrada y A. Equihua (eds.). Entomología mexicana. Sociedad Mexicana de Entomología, México.

Romero-López, A.A., R. Arzuffi \& M.A. Morón. 2005. Feromonas y atrayentes sexuales de coleópteros Melolonthidae de importancia agrícola. Folia Entomológica Mexicana, 44: 233-245.

Ruther, J., A. Reinecke, T. Tolasch \& M. Hilker. 2001. Make love not war: a common arthropod defense compound as sex pheromone in the forest cockchafer Melolontha hippocastani. Oecología, 128: 44-47.

Tada, S. \& W.S. Leal. 1997. Localization and morphology of the sex pheromone glands in scarab beetles (Coleoptera: Rutelinae, Melolonthinae). Journal of Chemical Ecology, 23: 903-915. 